\title{
Some Strong Convergence Theorems for Asymptotically almost Negatively Associated Random Variables
}

\author{
Haiwu Huang ${ }^{1,2,3, *}$, Xiongtao $\mathrm{Wu}^{1}$, Yanchun $\mathrm{Yi}^{1}$ \\ ${ }^{1}$ College of Mathematics and Statistics, Hengyang Normal University, Hengyang, PR China \\ ${ }^{2}$ College of Science, Guilin University of Technology, Guilin, PR China \\ ${ }^{3}$ Hunan Provincial Key Laboratory of Intelligent Information Processing and Application, Hengyang, PR China \\ *Corresponding author
}

\begin{abstract}
In this work, the complete moment convergence and $L_{p}$ convergence for asymptotically almost negatively associated (AANA, in short) random variables are investigated. As an application, the complete convergence theorem for weighted sums of AANA random variables is obtained. These theorems obtained extend and improve some earlier results.
\end{abstract}

Keywords: AANA random variables, complete moment convergence, $L_{p}$ convergence, weighted sums

Cite This Article: Haiwu Huang, Xiongtao Wu, and Yanchun Yi, "Some Strong Convergence Theorems for Asymptotically almost Negatively Associated Random Variables.” American Journal of Applied Mathematics and Statistics, vol. 5, no. 3 (2017): 106-111. doi: 10.12691/ajams-5-3-4.

\section{Introduction}

Definition 1.1 A finite collection of random variables $X_{1}, X_{2}, \ldots, X_{n}$ is said to be negatively associated (NA, in short) if for every pair of disjoint subsets $A_{1}$ and $A_{2}$ of $\{1,2, \ldots, n\}$,

$$
\operatorname{Cov}\left(f_{1}\left(X_{i}: i \in A_{1}\right), f_{2}\left(X_{j}: i \in A_{2}\right)\right) \leq 0
$$

whenever $f_{1}$ and $f_{2}$ are any real coordinatewise nondecreasing functions such that this covariance exists. An infinite sequence $\left\{X_{n} ; n \geq 1\right\}$ of random variables is said to be NA if for every finite sub-collection is NA.

The concept of NA was introduced by Joag-Dev and Proschan [4], and its probability limit properties have aroused wide interest because of their numerous applications in reliability theory, percolation theory and multivariate statistical analysis. By inspecting the proof of maximal inequality for NA random variables in Matula [7], one can also allow negative correlations provided they are small. Primarily motivated by this, Chandra and Ghosal $[1,2]$ introduced the following dependence.

Definition 1.2 A sequence $\left\{X_{n} ; n \geq 1\right\}$ of random variables is called AANA if there exists a nonnegative sequence $q(n) \rightarrow 0$ as $n \rightarrow \infty$ such that

$$
\begin{aligned}
& \operatorname{Cov}\left(f_{1}\left(X_{n}\right), f_{2}\left(X_{n+1}, \ldots, X_{n+k}\right)\right) \\
& \leq q(n)\left(\operatorname{Var}\left(f_{1}\left(X_{n}\right)\right) \operatorname{Var}\left(f_{2}\left(X_{n+1}, \ldots, X_{n+k}\right)\right)\right)^{1 / 2},
\end{aligned}
$$

for all $n \geq 1, \quad k \geq 1$, and for all coordinatewise nondecreasing continuous functions $f_{1}$ and $f_{2}$ whenever the variances exist.

The family of AANA sequence contains NA (in particular, independent) sequence (with $q(n)=0, n \geq 1$ ) and some more sequences of random variables which are not much deviated from being NA. Chandra and Ghosal [1] once pointed out that NA implies AANA, but AANA does not imply NA. Namely, AANA is much weaker than NA. Since, NA has been applied to the reliability theory, multivariate statistical analysis and percolation theory, and attracted extensive attentions. Hence, extending the limit properties of NA random variables to the wider case of AANA random variables is highly desirable in the theory and applications.

For recent various results and applications of AANA random variables, we can refer to that Chandra and Ghosal [1] obtained the Kolmogorov type inequality and the strong law of large numbers of Marcinkiewicz-Zygmund; Chandra and Ghosal [2] established the almost sure convergence of weighted averages; Wang et al. [10] obtained the law of the iterated logarithm for product sums; Ko et al. [5] studied the Hájek-Rényi type inequality; Yuan and An [14] established some Rosenthal type inequalities; Yuan and Wu [15] studied the limiting behavior of the maximum of the partial sum under residual Cesàro alpha-integrability assumption; Wang et al. [11,12], Huang et al. [3] studied the complete convergence of weighted sums for arrays of rowwise AANA random variables and arrays of rowwise AANA random variables, respectively; Yang et al. [16] investigated the complete convergence of moving average process for AANA sequence; and Tang [9] studied the strong law 
of large numbers for general weighted sums, Shen and $\mathrm{Wu}$ [8] obtained some new complete convergence results and Feller-type weak law of large numbers, and so forth.

Recently, Liang and Su [6] obtained the following complete convergence result for weighted sums of NA random variables.

Theorem A Let $\left\{X_{n} ; n \geq 1\right\}$ be a sequence of NA random variables with $E X_{n}=0,\left\{a_{n i} ; 1 \leq i \leq n, n \geq 1\right\}$ be an array of real numbers such that $\sum_{i=1}^{n} a_{n i}^{2}=O\left(n^{u}\right)$ and $a_{n i}=O(1)$ for $0<u<\frac{2}{p}, p \geq 2$. If $\sup _{i \geq 1} E\left|X_{i}\right|^{p}<\infty$, then for $\forall \varepsilon>0$,

$$
\sum_{n=1}^{\infty} n^{-1} P\left(\max _{1 \leq j \leq n}\left|\sum_{i=1}^{j} a_{n i} X_{i}\right|>\varepsilon n^{1 / p}\right)<\infty .
$$

Wang et al. [13] extended the result of Liang and Su [6] to AANA random variables as follows.

Theorem B Let $1 \leq r \leq 2,\left\{X_{n} ; n \geq 1\right\}$ be a sequence of AANA random variables with $E X_{n}<\infty$ and $E\left|X_{n}\right|^{r}<\infty$ for $n \geq 1$. Let $\left\{a_{n i} ; 1 \leq i \leq n, n \geq 1\right\}$ be an array of real numbers satisfying $\sum_{i=1}^{n}\left|a_{n i}\right|^{r} E\left|X_{i}\right|^{r}=O\left(n^{u}\right) \quad$ and $\sum_{n=1}^{\infty} q^{q / p}(n)<\infty \quad$ for $\quad$ some $\quad 0<u \leq \frac{2}{p} \quad$ and $p \in\left(3 \times 2^{k-1}, 4 \times 2^{k-1}\right)$, where integer number $k \geq 1$. Then for $\alpha r \geq 1$ and $\forall \varepsilon>0$,

$$
\sum_{n=1}^{\infty} n^{\alpha r-2} P\left(\max _{1 \leq j \leq n}\left|\sum_{i=1}^{j} \alpha_{n i} X_{i}\right| \geq \varepsilon n^{\alpha}\right)<\infty .
$$

This paper is motivated by Liang and Su [6], Wang et al. [13]. We further study the convergence properties for AANA random variables and establish the complete moment convergence theorem and $L_{p}(1<p<2)$ convergence theorem. As an application, the complete convergence and strong law of large numbers for weighted sums of AANA random variables are obtained. The obtained results extend and improve the above Theorem A and Theorem B.

The structure of this paper is as follows. In Section 2 , some important lemmas are firstly provided and the complete moment convergence theorem for AANA random variables is presented. In Section 3 , the $L_{p}$ convergence theorem is provided.

Throughout this paper, let $\left\{X_{n} ; n \geq 1\right\}$ be a sequence of AANA random variables with the mixing coefficients $\{q(n) ; n \geq 1\}$. Let $I(A)$ be the indicator function of the set $A$. The symbol $C$ will denote a positive constant which is not necessarily the same one in each appearance, $a_{n}=O\left(b_{n}\right)$ will stand for $a_{n} \leq C b_{n}$.

\section{Complete Moment Convergence}

In order to prove our main results, the following lemmas are needed.

Lemma 2.1 (Yuan and An [14]) Let $\left\{X_{n} ; n \geq 1\right\}$ be a sequence of AANA random variables with the mixing coefficients $\{q(n) ; n \geq 1\}, \quad f_{1}, f_{2}, \ldots, f_{n} \quad$ fn $\quad$ be all nondecreasing (or all nonincreasing) continuous functions, then $\left\{f_{n}\left(X_{n}\right) ; n \geq 1\right\}$ is still a sequence of AANA random variables with the mixing coefficients $\{q(n) ; n \geq 1\}$.

$$
E\left(\max _{1 \leq k \leq n}\left|\sum_{i=1}^{k} X_{i}\right|^{p}\right) \leq C \sum_{i=1}^{n} E\left|X_{i}\right|^{p}
$$

Theorem 2.1 Let $\left\{X_{n} ; n \geq 1\right\}$ be a sequence of AANA random variables with $\sum_{n=1}^{\infty} q^{2}(n)<\infty$ and $E X_{n}=0$ for all $n \geq 1$. Let $\left\{a_{n} ; n \geq 1\right\}$ be a sequence of positive real numbers. For some constant $\lambda>0$ and $1 \leq q<2$, if

$$
\begin{aligned}
& \sum_{n=1}^{\infty} a_{n} \sum_{i=1}^{n} E\left|X_{i}\right|^{q} I\left(\left|X_{i}\right|>\lambda\right)<\infty, \\
& \sum_{n=1}^{\infty} a_{n} \sum_{i=1}^{n} E\left|X_{i}\right|^{2} I\left(\left|X_{i}\right|>\lambda\right)<\infty, \\
& \sum_{n=1}^{\infty} E\left|X_{i}\right| I\left(\left|X_{i}\right|>\lambda\right) \rightarrow 0 \text { as } n<\infty .
\end{aligned}
$$

Then for $\forall \varepsilon>0$,

$$
\sum_{n=1}^{\infty} a_{n} E\left(\max _{1 \leq j \leq n}\left|\sum_{i=1}^{j} X_{i}\right|-\varepsilon\right)_{+}^{q}<\infty
$$

Theorem 2.2 Let $\left\{X_{n} ; n \geq 1\right\}$ be a sequence of AANA random variables with $\sum_{n=1}^{\infty} q^{2}(n)<\infty$ and $E X_{n}=0$, $E\left|X_{n}\right|^{p}<\infty$ for $1 \leq q<p<2$, all $n \geq 1$. Let $\left\{a_{n i} ; 1 \leq i \leq n, n \geq 1\right\}$ be an array of real numbers such that $\sum_{n=1}^{n}\left|a_{n i}\right|^{p} E\left|X_{i}\right|^{p}=O\left(n^{u}\right)$ for $0<u<1$ as $n \rightarrow \infty$. Then for $\alpha p \geq 1$ and $\forall \varepsilon>0$,

$$
\sum_{n=1}^{\infty} n^{\alpha p-2-\alpha q} E\left(\max _{1 \leq j \leq n}\left|\sum_{i=1}^{j} a_{n i} X_{i}\right|-\varepsilon n^{\alpha}\right)_{+}^{q}<\infty .
$$

Corollary 2.1 Under the conditions of Theorem 2.2, then

$$
\sum_{n=1}^{\infty} n^{\alpha p-2} P\left(\max _{1 \leq j \leq n}\left|\sum_{i=1}^{j} a_{n i} X_{i}\right|>\varepsilon n^{\alpha}\right)<\infty .
$$


Proof of Theorem 2.1 For $i \geq 1$, define

$$
\begin{aligned}
Y_{i} & =-\lambda I\left(X_{i}<-\lambda\right)+X_{i} I\left(\left|X_{i}\right| \leq \lambda\right)+\lambda I\left(X_{i}>\lambda\right), \\
& Z_{i}=X_{i}-Y_{i} \\
& =\left(X_{i}-\lambda\right) I\left(X_{i}>\lambda\right)+\left(X_{i}+\lambda\right) I\left(X_{i}<-\lambda\right) .
\end{aligned}
$$

From Lemma 2.1, the sequences of $\left\{Y_{i} ; i \geq 1\right\}$ and $\left\{Z_{i} ; i \geq 1\right\}$ are still AANA random variables. For $\forall \varepsilon>0$, then

$$
\begin{aligned}
& \sum_{n=1}^{\infty} a_{n} E\left(\max _{1 \leq j \leq n}\left|\sum_{i=1}^{j} X_{i}\right|-\varepsilon\right)_{+}^{q} \\
& =\sum_{n=1}^{\infty} a_{n} \int_{0}^{\infty} P\left(\left(\max _{1 \leq j \leq n}\left|\sum_{i=1}^{j} X_{i}\right|-\varepsilon\right)>s^{1 / q}\right) d s \\
& =\sum_{n=1}^{\infty} a_{n} \int_{0}^{\lambda^{q}} P\left(\max _{1 \leq j \leq n}\left|\sum_{i=1}^{j} X_{i}\right|>s^{1 / q}+\varepsilon\right) d s \\
& +\sum_{n=1}^{\infty} a_{n} \int_{\lambda}^{\infty} P\left(\max _{1 \leq j \leq n}\left|\sum_{i=1}^{j} X_{i}\right|>s^{1 / q}+\varepsilon\right) d s \\
& \leq C \lambda^{q} \sum_{n=1}^{\infty} a_{n} P\left(\max _{1 \leq j \leq n}\left|\sum_{i=1}^{j} X_{i}\right|>\varepsilon\right) \\
& +C \sum_{n=1}^{\infty} a_{n} \int_{\lambda^{q}}^{\infty} P\left(\max _{1 \leq j \leq n}\left|\sum_{i=1}^{j} X_{i}\right|>s^{1 / q}\right) d s \\
& \Delta I_{1}+I_{2} .
\end{aligned}
$$

To prove (2.5), it needs only to show that $I_{1}<\infty$, and $I_{2}<\infty$. When $\left|X_{i}\right| \leq \lambda, X_{i}=Y_{i}$, for $\forall \varepsilon>0$,

$$
\begin{aligned}
& P\left(\max _{1 \leq j \leq n}\left|\sum_{i=1}^{j} X_{i}\right|>\varepsilon\right) \\
& \leq P\left(\max _{1 \leq j \leq n}\left|\sum_{i=1}^{j} X_{i}\right|>\varepsilon, \bigcup_{i=1}^{n}\left(\left|X_{i}\right|>\lambda\right)\right) \\
& \quad+P\left(\max _{1 \leq j \leq n}\left|\sum_{i=1}^{j} X_{i}\right|>\varepsilon, \bigcap_{i=1}^{n}\left(\left|X_{i}\right| \leq \lambda\right)\right) \\
& \leq C \sum_{i=1}^{j} P\left(\left|X_{i}\right|>\lambda\right)+C P\left(\max _{1 \leq j \leq n}\left|\sum_{i=1}^{j} Y_{i}\right|>\varepsilon\right) .
\end{aligned}
$$

It follows from (2.2) that

$$
\begin{aligned}
& \lambda^{q} \sum_{n=1}^{\infty} a_{n} \sum_{i=1}^{n} P\left(\left|X_{i}\right|>\lambda\right)=\lambda^{q} \sum_{n=1}^{\infty} a_{n} \sum_{i=1}^{n} E I\left(\left|X_{i}\right|>\lambda\right) \\
& \leq C \lambda^{q} \sum_{n=1}^{\infty} a_{n} \sum_{i=1}^{n} E \frac{\left|X_{i}\right|^{q}}{\lambda^{q}} I\left(\left|X_{i}\right|>\lambda\right) \\
& =C \sum_{n=1}^{\infty} a_{n} \sum_{i=1}^{n} E\left|X_{i}\right|^{q} I\left(\left|X_{i}\right|>\lambda\right)<\infty .
\end{aligned}
$$

Note that $\left|Z_{i}\right| \leq\left|X_{i}\right| I\left(\left|X_{i}\right|>\lambda\right)$, it follows from $E X_{i}=0, \quad X_{i}=Z_{i}+Y_{i}$ and (2.4) that

$$
\begin{aligned}
& \max _{1 \leq j \leq n}\left|\sum_{i=1}^{j} E Y_{i}\right|=\max _{1 \leq j \leq n}\left|\sum_{i=1}^{j} E Z_{i}\right| \\
& \leq \sum_{i=1}^{n} E\left|X_{i}\right| I\left(\left|X_{i}\right|>\lambda\right) \rightarrow 0 \text { as } n \rightarrow \infty .
\end{aligned}
$$

By Markov inequality, (2.1) and $c_{r}$ inequality, we can obtain that

$$
\begin{aligned}
& \lambda^{q} \sum_{n=1}^{\infty} a_{n} P\left(\max _{1 \leq j \leq n}\left|\sum_{i=1}^{j} Y_{i}\right|>\varepsilon\right) \\
& \leq C \lambda^{q} \sum_{n=1}^{\infty} a_{n} \sum_{n=1}^{n} E\left|Y_{i}-E Y_{i}\right|^{2} \\
& \leq C \sum_{n=1}^{\infty} a_{n} \sum_{n=1}^{n} E\left|X_{i}\right|^{2} I\left(\left|X_{i}\right| \leq \lambda\right) \\
& +C \sum_{n=1}^{\infty} a_{n} \sum_{n=1}^{n} P\left(\left|X_{i}\right|>\lambda\right) \\
& \leq C \sum_{n=1}^{\infty} a_{n} \sum_{n=1}^{n} E\left|X_{i}\right|^{2} I\left(\left|X_{i}\right| \leq \lambda\right) \\
& +C \sum_{n=1}^{\infty} a_{n} \sum_{n=1}^{n} E\left|X_{i}\right|^{q} I\left(\left|X_{i}\right|>\lambda\right) \\
& <\infty .
\end{aligned}
$$

Hence, the desired result of $I_{1}<\infty$ follows from (2.10) immediately.

Secondly, we will show that $I_{2}<\infty$. For $s \geq \lambda^{q}$, define that

$$
\begin{aligned}
Y_{i}^{(')}= & -s^{1 / q} I\left(X_{i}<-s^{1 / q}\right)+X_{i} I\left(\left|X_{i}\right| \leq s^{1 / q}\right) \\
& +s^{1 / q} I\left(X_{i}>-s^{1 / q}\right), \\
Z_{i}^{(')}= & X_{i}-Y_{i}^{(')}=\left(X_{i}-s^{1 / q}\right) I\left(X_{i}<s^{1 / q}\right) \\
& +\left(X_{i}+s^{1 / q}\right) I\left(X_{i}<-s^{1 / q}\right) .
\end{aligned}
$$

By the similar argument as the proof of (2.9), we can obtain that

$$
\begin{aligned}
& P\left(\max _{1 \leq j \leq n}\left|\sum_{i=1}^{j} X_{i}\right|>s^{1 / q}\right) \\
& \leq C \sum_{i=1}^{n} P\left(\left|X_{i}\right|>s^{1 / q}\right)+C P\left(\max _{1 \leq j \leq n}\left|\sum_{i=1}^{j} Y_{i}^{(')}\right|>s^{1 / q}\right) .
\end{aligned}
$$

By (2.2), it follows that

$$
\begin{aligned}
& C \sum_{n=1}^{\infty} a_{n} \sum_{i=1}^{n} \int_{\lambda^{q}}^{\infty} P\left(\left|X_{i}\right|>s^{1 / q}\right) d s \\
& \leq C \sum_{n=1}^{\infty} a_{n} \sum_{i=1}^{n} E\left|X_{i}\right|^{q} I\left(\left|X_{i}\right|>\lambda\right)<\infty .
\end{aligned}
$$


Note that $\left|Z_{i}^{(')}\right| \leq\left|X_{i}\right| I\left(\left|X_{i}\right|>s^{1 / q}\right)$, it follows from $E X_{i}=0, Z_{i}^{(')}=X_{i}-Y_{i}^{(')}$ and (2.4) that

$$
\begin{aligned}
& \max _{s \geq \lambda^{q}} s^{-1 / q} \max _{1 \leq j \leq n}\left|\sum_{i=1}^{j} E Y_{i}^{(')}\right|=\max _{s \geq \lambda^{q}} s^{-1 / q} \max _{1 \leq j \leq n}\left|\sum_{i=1}^{j} E Z_{i}^{(')}\right| \\
& \leq \max _{s \geq \lambda^{q}} s^{-1 / q} \sum_{I=1}^{n} E\left|X_{i}\right| I\left(\left|X_{i}\right|>s^{1 / q}\right) \\
& \leq \lambda^{-1} \sum_{i=1}^{n} E\left|X_{i}\right| I\left(\left|X_{i}\right|>s^{1 / q}\right) \rightarrow 0 \text { as } n \rightarrow \infty,
\end{aligned}
$$

which implies

$$
\begin{aligned}
& P\left(\max _{1 \leq j \leq n}\left|\sum_{i=1}^{j} Y_{i}^{(')}\right|>s^{1 / q}\right) \\
& \leq P\left(\max _{1 \leq j \leq n}\left|\sum_{i=1}^{j}\left(Y_{i}^{(')}-E Y_{i}^{(')}\right)\right|>\frac{s^{1 / q}}{2}\right) \\
& \leq C s^{-2 / q} E\left(\max _{1 \leq j \leq n}\left|\sum_{i=1}^{j}\left(Y_{i}^{(')}-E Y_{i}^{(')}\right)\right|^{2}\right) \\
& \leq C s^{-2 / q} \sum_{i=1}^{n}\left|E\left(Y_{i}^{(')}-E Y_{i}^{(')}\right)\right|^{2} \\
& \leq C s^{-2 / q} \sum_{i=1}^{n}\left|X_{i}\right|^{2} I\left(\left|X_{i}\right| \leq s^{1 / q}\right) \\
& +C \sum_{i=1}^{n} P\left(\left|X_{i}\right|>s^{1 / q}\right) .
\end{aligned}
$$

For convenience, let $K=|\lambda|+1$, denote

$$
\begin{aligned}
& \nabla=\sum_{n=1}^{\Delta} a_{n} \sum_{i=1}^{n} \int_{\lambda}^{\infty} s^{-2 / q} E\left|X_{i}\right|^{2} I\left(\left|X_{i}\right| \leq s^{1 / q}\right) d s \\
& \leq \sum_{n=1}^{\infty} a_{n} \sum_{i=1}^{n} \int_{\lambda}^{\infty} s^{-2 / q} E\left|X_{i}\right|^{2} I\left(\left|X_{i}\right| \leq K\right) d s \\
& +\sum_{n=1}^{\infty} a_{n} \sum_{i=1}^{n} \int_{\lambda}^{\infty} s^{-2 / q} E\left|X_{i}\right|^{2} I\left(K<\left|X_{i}\right| \leq s^{1 / q}\right) d s .
\end{aligned}
$$

Hence,

$$
\begin{aligned}
& \nabla_{1} \stackrel{\Delta}{=} \sum_{n=1}^{\infty} a_{n} \sum_{i=1}^{n} \int_{\lambda}^{\infty} q^{-2 / q} E\left|X_{i}\right|^{2} I\left(\left|X_{i}\right| \leq K\right) d s \\
& \leq C \sum_{n=1}^{\infty} a_{n} \sum_{i=1}^{n} E\left|X_{i}\right|^{2} I\left(\left|X_{i}\right| \leq K\right) \\
& =C \sum_{n=1}^{\infty} a_{n} \sum_{i=1}^{n} E\left|X_{i}\right|^{2} I\left(\left|X_{i}\right| \leq \lambda\right) \\
& +C \sum_{n=1}^{\infty} a_{n} \sum_{i=1}^{n} E\left|X_{i}\right|^{2} I\left(\lambda<\left|X_{i}\right| \leq K\right)
\end{aligned}
$$

$$
\begin{aligned}
& \leq C \sum_{n=1}^{\infty} a_{n} \sum_{i=1}^{n} E\left|X_{i}\right|^{2} I\left(\left|X_{i}\right| \leq \lambda\right) \\
& +C K^{2-q} \sum_{n=1}^{\infty} a_{n} \sum_{i=1}^{n} E\left|X_{i}\right|^{q} I\left(\left|X_{i}\right|>\lambda\right) \\
& <\infty .
\end{aligned}
$$

Let $s=u^{q}$, it follows from $1 \leq q<2, K>\lambda$ and (2.2) that

$$
\begin{aligned}
& \nabla_{2}=\sum_{i=1}^{\Delta} \int_{\lambda}^{\infty} s^{-2 / q} E\left|X_{i}\right|^{2} I\left(K<\left|X_{i}\right| \leq s^{1 / q}\right) d s \\
& =\sum_{i=1}^{n} \int_{\lambda}^{K^{q}} s^{-2 / q} E\left|X_{i}\right|^{2} I\left(K<\left|X_{i}\right| \leq s^{1 / q}\right) d s \\
& +\sum_{i=1}^{n} \int_{K}^{\infty} s^{-2 / q} E\left|X_{i}\right|^{2} I\left(K<\left|X_{i}\right| \leq s^{1 / q}\right) d s \\
& \leq C \sum_{i=1}^{n} \int_{K}^{\infty} u^{q-3} E\left|X_{i}\right|^{2} I\left(K<\left|X_{i}\right| \leq u\right) d u \\
& =C \sum_{i=1}^{n} \sum_{m=K}^{\infty} \int_{m}^{m+1} u^{q-3} E\left|X_{i}\right|^{2} I\left(K<\left|X_{i}\right| \leq u\right) d u \\
& \leq C \sum_{i=1}^{n} \sum_{m=K}^{\infty} m^{q-3} E\left|X_{i}\right|^{2} I\left(K<\left|X_{i}\right| \leq m+1\right) \\
& =C \sum_{i=1}^{n} \sum_{m=K}^{\infty} m^{q-3} \sum_{k=K}^{m} E\left|X_{i}\right|^{2} I\left(k<\left|X_{i}\right| \leq k+1\right) \\
& \leq C \sum_{i=1}^{n} \sum_{k=K}^{\infty} k^{q-2} E\left|X_{i}\right|^{2} I\left(k<\left|X_{i}\right| \leq k+1\right) \\
& \leq C \sum_{i=1}^{n} E\left|X_{i}\right|^{q} I\left(\left|X_{i}\right|>\lambda\right) .
\end{aligned}
$$

Hence, the desired result of $I_{2}<\infty$ follows from (2.2) and the above statements immediately. The proof of Theorem 2.1 is completed.

Proof of Theorem 2.2 Let $a_{n}=n^{\alpha p-2}$ and $X_{i}=\frac{a_{n i} X_{i}}{n^{\alpha}}$ in Theorem 2.1, then

$$
\begin{aligned}
& \sum_{n=1}^{\infty} a_{n} \sum_{i=1}^{n} E\left|X_{i}\right|^{q} I\left(\left|X_{i}\right|>\lambda\right) \\
& =\sum_{n=1}^{\infty} n^{\alpha p-2} \sum_{i=1}^{n} E\left|\frac{a_{n i} X_{i}}{n^{\alpha}}\right|^{q} I\left(\left|a_{n i} X_{i}\right|>\lambda n^{\alpha}\right) \\
& =\sum_{n=1}^{\infty} n^{\alpha p-\alpha q-2} \sum_{i=1}^{n} E\left|a_{n i} X_{i}\right|^{q} I\left(\left|a_{n i} X_{i}\right|>\lambda n^{\alpha}\right) \\
& \leq \lambda^{q-p} \sum_{n=1}^{\infty} n^{-2} \sum_{i=1}^{n}\left|a_{n i}\right|^{p} E\left|X_{i}\right|^{p} I\left(\left|a_{n i} X_{i}\right|>\lambda n^{\alpha}\right) \\
& \leq C \lambda^{q-p} \sum_{n=1}^{\infty} n^{-2+u}<\infty .
\end{aligned}
$$




$$
\begin{aligned}
& \sum_{n=1}^{\infty} a_{n} \sum_{i=1}^{n} E\left|X_{k}\right|^{2} I\left(\left|X_{k}\right|>\lambda\right) \\
& =\sum_{n=1}^{\infty} n^{\alpha p-2} \sum_{i=1}^{n} E\left|\frac{a_{n i} X_{i}}{n^{\alpha}}\right|^{2} I\left(\left|a_{n i} X_{i}\right| \leq \lambda n^{\alpha}\right) \\
& \leq \lambda^{2-p} \sum_{n=1}^{\infty} n^{-2} \sum_{i=1}^{n}\left|a_{n i}\right|^{p} E\left|X_{i}\right|^{p} I\left(\left|a_{n i} X_{i}\right| \leq \lambda n^{\alpha}\right) \\
& \leq C \lambda^{2-p} \sum_{n=1}^{\infty} n^{-2+u}<\infty .
\end{aligned}
$$

It follows from $E X_{n}=0$ and $\sum_{n=1}^{\infty}\left|a_{n i}\right|^{p} E\left|X_{i}\right|^{p}=O\left(n^{u}\right)$ that

$$
\begin{aligned}
& \sum_{n=1}^{\infty} E\left|X_{i}\right| I\left(\left|X_{i}\right|>\lambda\right) \\
& =n^{-\alpha} \sum_{n=1}^{\infty} E\left|a_{n i} X_{i}\right| I\left(\left|a_{n i} X_{i}\right|>\lambda n^{\alpha}\right) \\
& \leq \lambda^{1-p} n^{-\alpha p} \sum_{n=1}^{\infty}\left|a_{n i}\right|^{r} E\left|X_{i}\right|^{r} I\left(\left|a_{n i} X_{i}\right|>\lambda n^{\alpha}\right) \\
& \leq C \lambda^{1-p} n^{u-\alpha p} \rightarrow 0 .
\end{aligned}
$$

The proof of Theorem 2.2 is completed.

\section{3. $L_{p}$ Convergence}

In this section, we will state the $L_{p}$ convergence under some conditions.

Theorem 3.1 Let $\left\{X_{n} ; n \geq 1\right\}$ be a sequence of AANA random variables with $\sum_{n=1}^{\infty} q^{2}(n)<\infty$ and $E X_{n}=0$. For $1 \leq p<2$, suppose that for some $\lambda>0$

$$
\begin{aligned}
& \sum_{i=1}^{n}\left|E X_{i}\right|^{p} I\left(\left|X_{i}\right|>\lambda\right) \rightarrow 0 \text { as } n \rightarrow \infty, \\
& \sum_{i=1}^{n} E\left|X_{i}\right|^{2} I\left(\left|X_{i}\right| \leq \lambda\right) \rightarrow 0 \text { as } n \rightarrow \infty .
\end{aligned}
$$

Then,

$$
\max _{1 \leq j \leq n} \mid \sum_{i=1}^{j} X_{i} \stackrel{{ }_{p}}{\longrightarrow} 0 \text { as } n \rightarrow \infty .
$$

Proof of Theorem 3.1 For $\forall \varepsilon>0$, we use the same notations of Theorem 2.1,

$$
\begin{gathered}
Y_{i}=-\lambda I\left(X_{i}<-\lambda\right)+X_{i} I\left(\left|X_{i}\right| \leq \lambda\right)+\lambda I\left(X_{i}>\lambda\right), \\
Z_{i}=X_{i}-Y_{i} \\
=\left(X_{i}-\lambda\right) I\left(X_{i}>\lambda\right)+\left(X_{i}+\lambda\right) I\left(X_{i}<-\lambda\right) .
\end{gathered}
$$

It follows from $c_{r}$ inequality and $E X_{n}=0$ that

$$
\begin{aligned}
& E\left(\max _{1 \leq j \leq n}\left|\sum_{i=1}^{j} X_{i}\right|\right)^{p} \\
& \leq E\left(\max _{1 \leq j \leq n}\left|\sum_{i=1}^{j}\left(Z_{i}-E Z_{i}\right)\right|\right)^{p}+E\left(\max _{1 \leq j \leq n}\left|\sum_{i=1}^{j}\left(Y_{i}-E Y_{i}\right)\right|\right)^{p} \\
& \leq E\left(\max _{1 \leq j \leq n}\left|\sum_{i=1}^{j}\left(Z_{i}-E Z_{i}\right)\right|\right)^{p} \\
& +\left(\max _{1 \leq j \leq n}\left|\sum_{i=1}^{j}\left(Y_{i}-E Y_{i}\right)\right|^{p}\right)^{P / 2} \\
& =J_{1}+J_{2} .
\end{aligned}
$$

Note that $\left|Z_{i}\right| \leq\left|X_{i}\right| I\left(\left|X_{i}\right|>\lambda\right)$, it follows from Lemma 2.2 and (3.1) that

$$
\begin{aligned}
& J_{1} \stackrel{\Delta}{=} E\left(\max _{1 \leq j \leq n}\left|\sum_{i=1}^{j}\left(Z_{i}-E Z_{i}\right)\right|\right)^{p} \\
& \leq C \sum_{i=1}^{n} E\left|Z_{i}-E Z_{i}\right|^{p} \\
& \leq C \sum_{i=1}^{n} E\left|Z_{i}\right|^{p} \\
& \leq C \sum_{i=1}^{n} E\left|X_{i}\right|^{p} I\left(\left|X_{i}\right|>\lambda\right) \rightarrow 0 \text { as } N \rightarrow \infty .
\end{aligned}
$$

By $c_{r}$ inequality, Lemma 2.2, (3.1) and (3.2), we can obtain that

$$
\begin{aligned}
& J_{2}^{\prime}=E\left(\max _{1 \leq j \leq n}\left|\sum_{i=1}^{j}\left(Y_{i}-E Y_{i}\right)\right|\right)^{2} \\
& \leq C \sum_{i=1}^{n} E\left|Y_{i}-E Y_{i}\right|^{2} \\
& \leq C \sum_{i=1}^{n} E\left|Y_{i}\right|^{2} \\
& \leq C \sum_{i=1}^{n} E\left|X_{i}\right|^{2} I\left(\left|X_{i}\right| \leq \lambda\right)+C \lambda^{2} \sum_{i=1}^{n} P\left(\left|X_{i}\right|>\lambda\right) \\
& \leq C \sum_{i=1}^{n} E\left|X_{i}\right|^{2} I\left(\left|X_{i}\right| \leq \lambda\right)+C \sum_{i=1}^{n} E\left|X_{i}\right|^{p}\left(\left|X_{i}\right|>\lambda\right) \rightarrow 0 .
\end{aligned}
$$

From (3.4) and (3.5), we can obtain $J_{1} \rightarrow 0$ and $J_{2} \rightarrow 0$ as $n \rightarrow \infty$. The proof of Theorem 3.1 is completed.

Take $X_{i}=X_{i} / a_{n}$ in Theorem 3.1, we can immediately obtain the following result.

Corollary 3.1 Let $\left\{X_{n} ; n \geq 1\right\}$ be a sequence of AANA random variables with $\sum_{n=1}^{\infty} q^{2}(n)<\infty$ and $E X_{n}=0$. For $1<p<2$, suppose that for some $\lambda>0$ 


$$
\begin{gathered}
a_{n}^{-p} \sum_{i=1}^{n} E\left|X_{i}\right|^{p} I\left(\left|X_{i}\right|>\lambda a_{n}\right) \rightarrow 0 \text { as } n \rightarrow \infty, \\
a_{n}^{-2} \sum_{i=1}^{n} E\left|X_{i}\right|^{2} I\left(\left|X_{i}\right| \leq \lambda a_{n}\right) \rightarrow 0 \text { as } n \rightarrow \infty .
\end{gathered}
$$

Then,

$$
a_{n}^{-2} \max _{1 \leq j \leq n}\left|\sum_{i=1}^{j} X_{i}\right| \stackrel{L_{p}}{\longrightarrow} 0 \text { as } n \rightarrow \infty .
$$

Corollary 3.2 Let $\left\{X_{n} ; n \geq 1\right\}$ be a sequence of AANA random variables with $\sum_{n=1}^{\infty} q^{2}(n)<\infty$ and $E X_{n}=0$. For $1 \leq p<2$, suppose that for some $\lambda>0$

$$
\sum_{i=1}^{n} P\left(\left|X_{i}\right|>\lambda\right) \rightarrow 0 \text { as } n \rightarrow \infty .
$$

Then (3.2) and (3.9) imply

$$
\max _{1 \leq j \leq n}\left|\sum_{i=1}^{j} X_{i}\right| \stackrel{P}{\longrightarrow} 0 \text { as } n \rightarrow \infty \text {. }
$$

Proof of Corollary 3.2 For $\forall \varepsilon>0$, we use the same notation of Theorem 2.1, It follows from $c_{r}$ inequality and $E X_{n}=0$ that

$$
\begin{aligned}
& P\left(\max _{1 \leq j \leq n}\left|\sum_{i=1}^{j} X_{i}\right|>2 \varepsilon\right) \\
& \leq P\left(\max _{1 \leq j \leq n}\left|\sum_{i=1}^{j}\left(Z_{i}-E Z_{i}\right)\right|>\varepsilon\right) \\
& +P\left(\max _{1 \leq j \leq n}\left|\sum_{i=1}^{j}\left(Y_{i}-E Y_{i}\right)\right|>\varepsilon\right) \\
& =K_{1}+K_{2} .
\end{aligned}
$$

By Markov inequality, Lemma 2.2, (3.9) and (3.2), we can obtain that

$$
\begin{aligned}
& K_{2} \leq C E\left(\max _{1 \leq j \leq n}\left|\sum_{i=1}^{j}\left(Y_{i}-E Y_{i}\right)\right|\right)^{2} \\
& \leq C \sum_{i=1}^{n} E\left|Y_{i}-E Y_{i}\right|^{2} \leq C \sum_{i=1}^{n} E\left|Y_{i}\right|^{2} \\
& \leq C \sum_{i=1}^{n} E\left|X_{i}\right|^{2} I\left(\left|X_{i}\right| \leq \lambda\right) \\
& +C \lambda^{2} \sum_{i=1}^{n} P\left(\left|X_{i}\right|>\lambda\right) \rightarrow 0 .
\end{aligned}
$$

Take into account the definition of $Z_{i}$ and (3.9), we can obtain that

$$
\begin{aligned}
K_{1} & \leq P\left(\exists j: 1 \leq j \leq n, \text { such that }\left|X_{i}\right|>\lambda\right) \\
& \leq \sum_{i=1}^{n} P\left(\left|X_{i}\right|>\lambda\right) \rightarrow 0 \text { as } n \rightarrow \infty .
\end{aligned}
$$

The proof of Corollary 3.2 is completed.

\section{Support}

This work is supported by the National Nature Science Foundation of China (11526085), the Humanities and Social Sciences Foundation for the Youth Scholars of Ministry of Education of China (15YJCZH066), the Science and Technology Plan Project of Hunan Province (2016TP1020), the Construct Program of the Key Discipline in Hunan Province.

\section{References}

[1] Chandra TK and Ghosal S. 1996a. Extensions of the strong law of large numbers of Marcinkiewicz and Zygmund for dependent variables. Acta Math. Hungar 71: 327-336.

[2] Chandra TK and Ghosal S. 1996b. The strong law of large numbers for weighted averages under dependence assumptions. J. Theor. Prob. 9: 797-809.

[3] Huang HW et al. On the strong convergence for weighted sums of asymptotically almost negatively associated random variables. Kybernetika 50: 393-407.

[4] Joag-Dev K and Proschan F. 1983. Negative association of random variables with applications. Ann. Stat. 11: 286-295.

[5] Ko MH et al. 2005. The H_ajeck-R_enyi inequality for the AANA random variables and its applications. Taiwanese Math. 9: 111-122.

[6] Liang HY and Su C. 1999. Complete convergence for weighted sums of NA sequences. Stat. Prob. Lett. 45: 85-95.

[7] Matula P. 1992. A note on the almost sure convergence of sums of negatively dependent random variables. Stat. Prob. Lett. 15: 209-213.

[8] Shen AT and Wu RC. 2013. Strong and weak convergence for asymptotically almost negatively associated random variables. Discrete Dyn. Nat. Soc. 2013, Article ID 235012, 7.

[9] Tang XF. 2013. Some strong laws of large numbers for weighted sums of asymptotically almost negatively associated random variables. J. Ineq. Appl. 2013.

[10] Wang YB et al. 2003. The strong law of large numbers and the law of the iterated logarithm for product sums of NA and AANA random variables. Southeast Asian Bull. Math. 27: 369-384.

[11] Wang XJ et al. 2012a. On complete convergence of weighted sums for arrays of rowwise asymptotically almost negatively associated random variables. Abst. Appl. Anal. 2012, Article ID 315138, 15.

[12] Wang XJ et al. 2011. Complete convergence for arrays of rowwise asymptotically almost negatively associated random variables. Discrete Dyn. Nat. Soc. 2011, Article ID 717126, 11.

[13] Wang XJ et al. 2010. Convergence properties for asymptotically almost negatively associated sequence. Discrete Dyn. Nat. Soc. 2010, Article ID 218380, 15 pages.

[14] Yuan DM and An J. 2009. Rosenthal type inequalities for asymptotically almost negatively associated random variables and applications. Sci. China. Ser A: Mathematics. 52: 1887-1904.

[15] Yuan DM and Wu XS. 2010. Limiting behavior of the maximum of the partial sum for asymptotically negatively associated random variables under residual Cesàro alpha-integrability assumption. J. Stat. Plan. Infer. 140: 2395-2402.

[16] Wang XJ et al. 2012b. On complete convergence of moving average process for AANA sequence. Discrete Dyn. Nat. Soc. 2012, Article ID 863931, 24. 